\title{
Shape-Selective Synthesis of Pentacene Macrocycles and the Effect of Geometry on Singlet Fission
}

\author{
Harrison M. Bergman, ${ }^{1}$ Gavin R. Kiel, ${ }^{1,+}$ Ryan J. Witzke, ${ }^{1,+}$ David P. Nenon, ${ }^{1,2}$ Adam M. Schwartzberg, ${ }^{2,3}$ Yi Liu, ${ }^{2,3}$ \\ and T. Don Tilley,* \\ 'Department of Chemistry, University of California, Berkeley, Berkeley, California 94720, United States \\ ${ }^{2}$ Material Sciences Division, Lawrence Berkeley National Laboratory, Berkeley, California 94720, United States \\ ${ }^{3}$ Molecular Foundry, Lawrence Berkeley National Lab, Berkeley, California 94720, United States \\ Supporting Information Placeholder
}

\begin{abstract}
Pentacene's extraordinary photophysical and electronic properties are highly dependent on intermolecular, through-space interactions. Macrocyclic arrangements of chromophores have been shown to provide a high level of control over these interactions, but few examples exist for pentacene due to inherent synthetic challenges. In this work, zirconocene-mediated alkyne coupling was used as a dynamic covalent $\mathrm{C}-\mathrm{C}$ bond forming reaction to synthesize two geometrically distinct, pentacene-containing macrocycles on a gram scale and in four or fewer steps. Both macrocycles undergo singlet fission in solution, with rates that differ by an order of magnitude while the rate of triplet recombination is approximately the same. This independent modulation of singlet and triplet decay rates is highly desirable for the design of efficient singlet fission materials. The dimeric macrocycle adopts a columnar packing motif in the solid state, with large void spaces between pentacene units of the crystal lattice.
\end{abstract}

Pentacene is one of the most studied polycyclic aromatic hydrocarbons (PAHs), largely because of its high charge mobility and reputation as a prototypical organic p-type semiconductor. ${ }^{1,2}$ However, more recently it has attracted interest for its ability to perform efficient, exothermic singlet fission (SF). ${ }^{3,4}$ This process, whereby one photon generates two triplet excitons, has garnered significant interest as a strategy to increase the efficiency of traditional inorganic solar cells, theoretically enabling them to break the Shockley-Quiesser limit of $29 \%$ for an ideal silicon cell. ${ }^{5,6}$

Due to the mechanistic complexity of this process, significant effort has gone into understanding the fundamental relationships between electronic coupling of chromophores and the resulting SF dynamics through the use of covalently linked synthetic model systems. ${ }^{7-22}$ These stud- ies have revealed considerable mechanistic detail and several reliable design rules for controlling the rate of singlet fission and the lifetime of the resulting triplets; however, these parameters are usually inversely related, and it is of ongoing interest to develop new strategies for their independent modulation, which has only recently been achieved via the energy cleft design reported by Pun et al. ${ }^{23}$

While the study of molecular model systems in solution is invaluable for developing fundamental understanding, technological applications demand efficient SF in the solid on significantly longer length scales. To this end, several elegant strategies have been developed, including crystal engineering, ${ }^{24,25}$ formation of liquid crystalline phases, ${ }^{26,27}$ and aggregation of chromophores on surfaces. ${ }^{28,29}$

Recently, shape persistent macrocycles have emerged as scaffolds capable of arranging chromophores across multiple length scales, making them interesting targets both as molecular model systems and for performance in the solidstate. ${ }^{30-33}$ The rigid, cyclic scaffold facilitates the intramolecular, through-space electronic coupling of chromophores while also inducing supramolecular assembly, generating aggregates in solution and crystalline materials with enhanced photophysical properties. ${ }^{34,35}$ For example, Nuckolls has shown that in certain cases, macrocyclic frameworks promote superior device performance as compared to acyclic counterparts. ${ }^{36}$ This diverse behavior for macrocycles, in conjunction with a strong fundamental understanding of singlet fission, should enable the design of pentacene-containing macrocycles with rich photophysical and supramolecular behavior.

To date there are only two reports of pentacenecontaining macrocycles, and a single macrocycle consisting of a fully conjugated backbone (Figure 1a). ${ }^{37,38}$ The results of Yoshizawa are of particular interest, as they illustrate the potential for macrocyclic frameworks to enforce interesting 
multi-chromophore interactions in a rigid trimeric scaffold that facilitates communication between three adjacent pentacene units, enabling efficient singlet fission in solution; however, these results relied on traditional macrocyclization methods which are associated with limited synthetic yields and scalability.

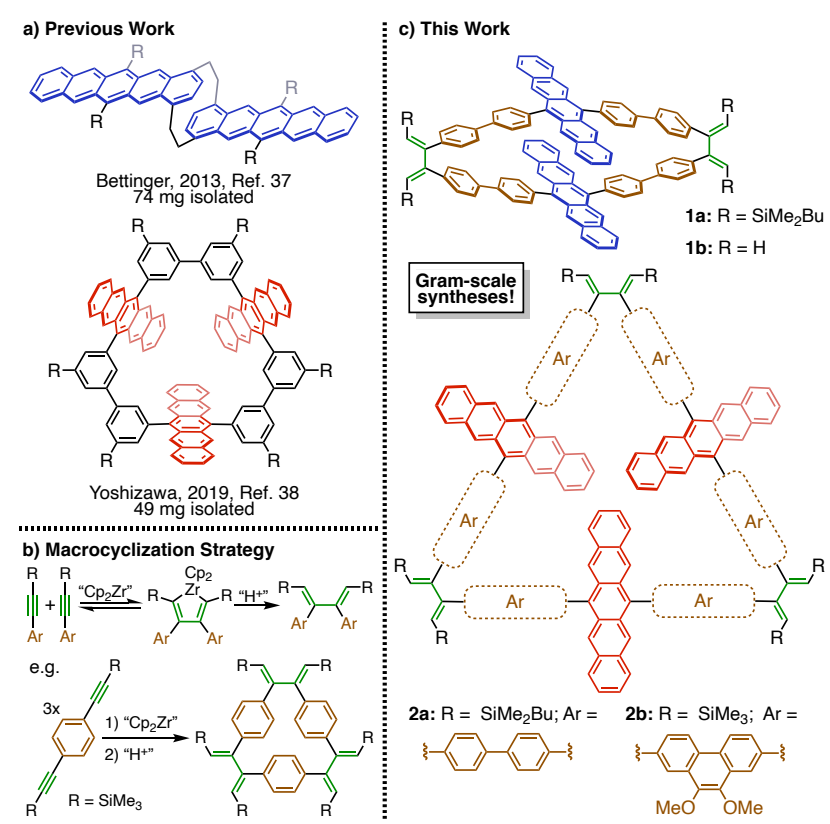

Figure 1. Depictions of a) previously synthesized pentacenecontaining macrocycles, b) the zirconocene mediated macrocyclization strategy, and c) macrocycles synthesized in this work.

The Tilley laboratory introduced the use of zirconocenemediated alkyne coupling in dynamic covalent chemistry (DCC) involving C-C bonds, for the synthesis of rigid macrocycles (Figure 1b) $\cdot{ }^{39}$ Although this method has been underutilized relative to alkyne and alkene metathesis, ${ }^{40-43}$ it is notable for its uniformly high yields, scalability, and the ability to rationally control macrocyclic structure. Thus, it seemed well suited for the scalable synthesis of pentacenecontaining macrocycles to study the effect of geometry on singlet fission dynamics and solid-state packing. As described below, this has been accomplished with selective syntheses of dimeric and trimeric pentacene macrocycles, using rational linker design, from commercially available materials in four or fewer steps (Figure 1c). Both macrocycles undergo singlet fission, with the rate of singlet fission modulated by the geometric constraints of the scaffold while triplet lifetime is significantly less perturbed. The dimeric macrocycle also displays unusual solid-state packing characterized by periodic void space between pentacene units.
Scheme 1. Synthetic Route for Non-selective Synthesis of Dimeric and Trimeric Macrocycles

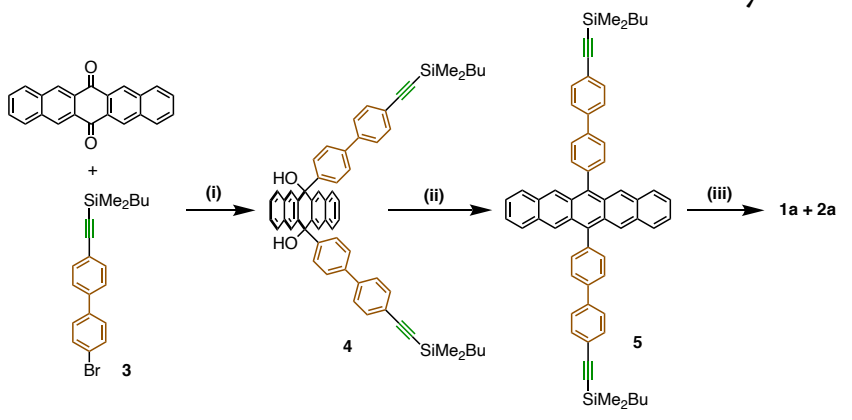

Reagents and conditions: (i) nBuLi, THF, $-78^{\circ} \mathrm{C}$ to $23^{\circ} \mathrm{C}$, $55 \%$; (ii) $\mathrm{NaI}, \mathrm{NaH}_{2} \mathrm{PO}_{2}, \mathrm{AcOH}, 120^{\circ} \mathrm{C}, 97 \%$; (iii) a) Rosenthal's Complex , Toluene, $60{ }^{\circ} \mathrm{C}$; b) $\mathrm{HCl}$, THF, $23{ }^{\circ} \mathrm{C}, 71 \%$.

Initial attempts to synthesize pentacene macrocycles focused on monomer 5 containing a simple, easily accessible biphenyl linker. In addition to its simplicity, the biphenyl linker was chosen based on this group's prior report synthesizing an anthracene macrocycle with phenylene linkers, ${ }^{44}$ and the previously unpublished synthesis of an anthracene dimer bearing linker 3 (Scheme S1). Monomer 5 was accessed via a two-fold nucleophilic addition of lithiated 4-bromo-4'-butyldimethylsilylethynyl-biphenyl across 6,13-pentacenequinone in $55 \%$ yield, followed by reductive aromatization of the resulting pentacene diol 4 in $97 \%$ yield (Scheme 1).

Monomer $\mathbf{5}$ was then subjected to zirconocene coupling conditions with Rosenthal's complex (7), 45,46 and demetalation with hydrochloric acid in situ furnished macrocyclic products as a mixture of the expected dimer $\mathbf{1 a}$ and trimeric macrocycle $2 a$ in a 47:53 ratio. This ratio could be modulated to some extent by varying concentration (Table S1), but selective synthesis was not achievable. Furthermore, these products were exceptionally difficult to separate by preparatory TLC (see SI), and amenable to isolation on only a very small scale. Thus, this route did not provide a scalable macrocyclization method, and a new synthetic strategy was adopted.

Given that zirconocene macrocyclizations of this type are reversible, the above ratio of products indicates that there is little thermodynamic preference for 1a vs. $\mathbf{2 a}$ under the reaction conditions. This implies that the biphenyl linker is fairly adaptable, in that it is flexible enough to form the predicted dimeric macrocycle but bending is apparently disfavored enough to make trimerization almost as favorable. It therefore seemed that a bent linker might strongly favor dimerization, while rigidification of the linear biphenyl unit should favor trimerization. 
Scheme 2. Selective Syntheses of Dimeric and Trimeric Macrocycles

a) Selective synthesis of dimeric pentacene macrocycle
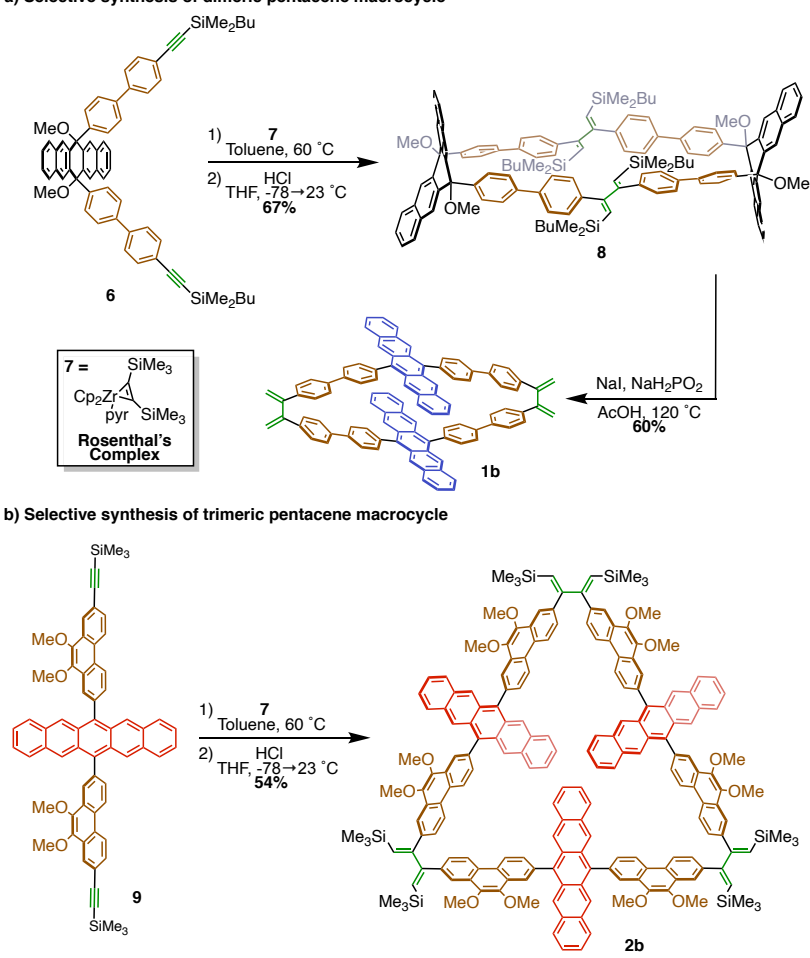

To test these conjectures, the protected cis-pentacene diol monomer 6 was targeted (Scheme 2a). Macrocyclization and demetallation of $\mathbf{6}$, under conditions similar to those described above, afforded dimeric macrocycle $\mathbf{8}$ as the sole well-defined product in $67 \%$ isolated yield. Importantly, this macrocyclization was performed on a 2.5 gram, facilitating large scale production of the final product. Macrocycle 8 was then subjected to reductive aromatization conditions as above to furnish the desired dimeric macrocycle $\mathbf{1 b}$ in $60 \%$ yield. The identity of this macrocycle was confirmed by ${ }^{1} \mathrm{H}$ NMR spectroscopy, MALDI, and $\mathrm{X}$-ray crystallography, all of which indicate that the silyl groups on the exocyclic dienes are cleaved under the reductive aromatization conditions.

To access the trimeric macrocycle, an analog of parent monomer 5 was synthesized bearing phenanthrene-based linkers, designed as "rigidified biphenyls" to act as braces that hinder bending. This monomer (9) was synthesized in three steps from pentacenequinone in a manner analogous to the synthesis of monomer $\mathbf{5}$ (Scheme S2). Macrocycle $\mathbf{2 b}$ was then produced as the sole well-defined product in $54 \%$ yield under analogous macrocyclization and demetallation conditions on gram scale (Scheme $\mathbf{2 b}$ ). The macrocycle's identity was confirmed by ${ }^{1} \mathrm{H}$ NMR spectroscopy and MALDI.
The crystal structure of dimer $\mathbf{1 b}$ displays several unexpected features. There is an unusually wide spacing of 7.6 $\AA$ A between the two pentacene units within the macrocycle, with two chloroform molecules (not pictured) as guests within the cavity (Figure 2a). This large inter-pentacene spacing is believed to be driven by packing forces in the solid state, suggested by comparison with the gas-phase optimized structure which shows a significantly reduced spacing of $5.2 \AA$ (Figure $\mathbf{2 b}$ ). This expansion of the macrocyclic backbone facilitates a unique columnar stacking motif along the $a$ crystallographic axis, driven by intermolecular $\pi-\pi$ stacking $(3.5 \AA)$ of pentacenes on adjacent macrocycles (Figure 2c). This introduction of periodic void space into the crystal lattice is unprecedented in pentacenes, the vast majority of which pack either as isolated dimers or in a continuous slip-stacked or herringbone fashion. ${ }^{25,47}$
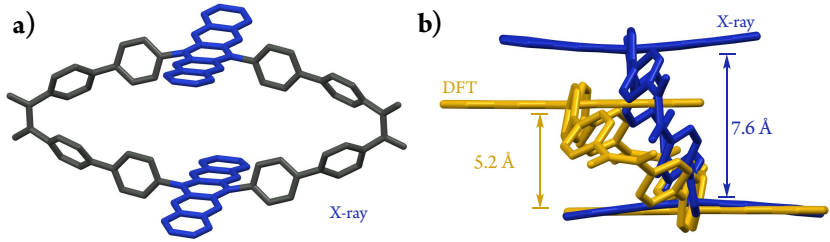

c)

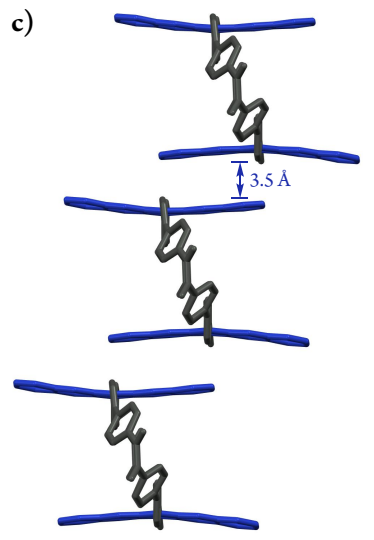

d)

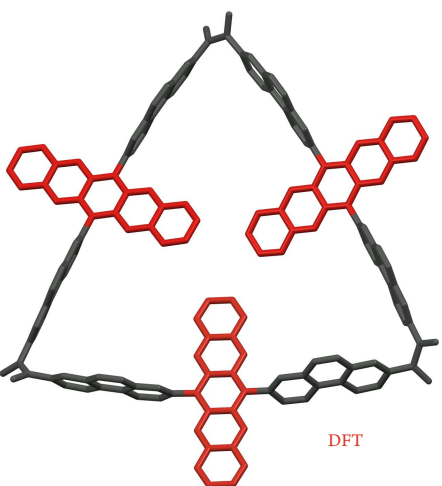

Figure 2. X-ray crystallographic structure of $\mathbf{1 b}$ and optimized (B3LYP/6-31G(d)) structures of $\mathbf{1 b}$ and $\mathbf{2} \mathbf{b}$ with hydrogen atoms and solvent omitted for clarity: a) front view of $\mathbf{1 b} ; \mathbf{b}$ ) comparison of optimized (yellow) and crystallographic (blue) structure of $\mathbf{1 b}$; $\mathbf{c}$ ) side view of crystal packing of $\mathbf{1 b}$; d) optimized structure of $\mathbf{2} \mathbf{b}$ with all sidechains omitted for clarity.

For $\mathbf{2 b}$, although crystals of a suitable size for X-ray crystallography were grown under various conditions, all were highly disordered and a reasonable structural model could not be obtained. In lieu of a crystallographic analysis, the structure of $\mathbf{2} \mathbf{b}$ was optimized by DFT at the B3LYP/6$31 G(d)$ level of theory. 


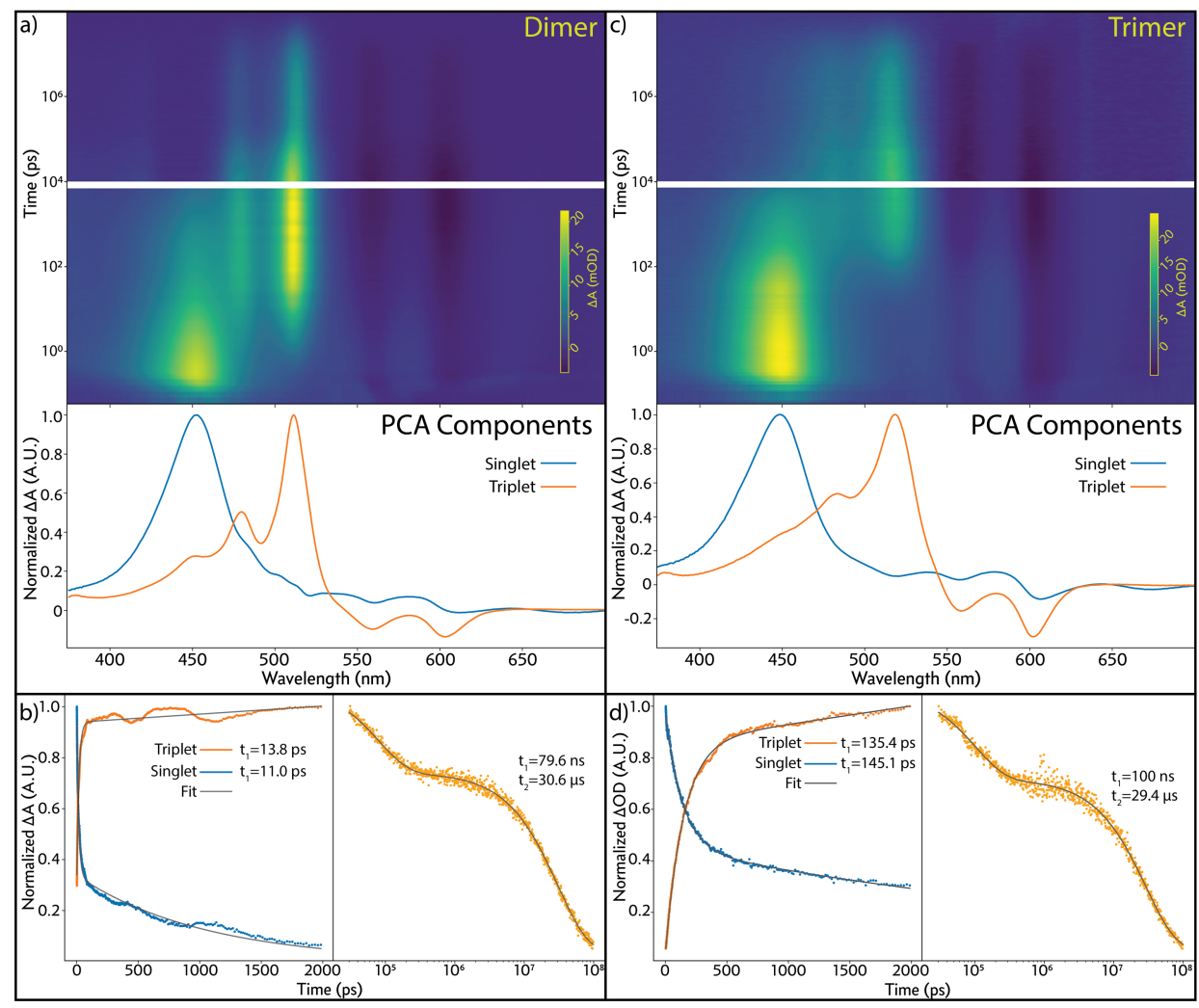

Figure 3. Transient absorption spectroscopy of $\mathbf{1 b}$ and $\mathbf{2 b}$. $\mathbf{a}$ and $\mathbf{c}$ : normalized $2 \mathrm{D}$ maps of transient absorption data for $\mathbf{1 b}$ and $\mathbf{2 b}$ respectively. Deconvoluted spectra of singlet (blue) and triplet (gold) species solved by principle component analysis; $\mathbf{b}$ and $\mathbf{d}$ : normalized kinetic traces of singlet and triplet populations on the picosecond timescale, and a triplet decay trace on the nanosecond scale.

Due to their distinct geometries, macrocycles $\mathbf{1 b}$ and $\mathbf{2 b}$ facilitate markedly different through-space interactions between their constituent pentacene units. Macrocycle $\mathbf{1 b}$ displays a pseudo slip-stacked arrangement, where there is a high degree of alignment between the acenes but a large inter-pentacene spacing that lowers effective $\pi$-overlap. Macrocycle 2b displays only weak edge-to-face interactions, but significantly closer inter-pentacene distances of 3.6-3.7 ̊.

Next, it was of interest to determine how these distinct geometries influenced photophysical and electronic properties. The relative orientation of the pentacene units was shown to have little effect on both steady state UV-Vis and fluorescence spectra (Table 1), with macrocycles $\mathbf{1 b}$ and 2b exhibiting almost identical behavior to each other and their respective monomers ( $\mathbf{5}$ and $\mathbf{8})$. Notably however, the photoluminescence quantum yields (PLQY) of the macrocycles were several times lower than those of their respective monomers. While low PLQY can potentially be attributed to the emergence of several non-radiative relaxation pathways, in pentacene systems this is often indicative of singlet fission. ${ }^{8,9}$
Table 1. Summary of photophysical properties of key macrocycles and their monomers.

\begin{tabular}{|c|c|c|c|c|c|}
\hline Compound & $\begin{array}{c}\lambda_{\text {abs }} \\
(\mathrm{nm})\end{array}$ & $\begin{array}{c}\lambda_{\mathrm{em}} \\
(\mathrm{nm})\end{array}$ & $\begin{array}{c}\text { PLQY } \\
(\%)\end{array}$ & $\begin{array}{c}\tau_{\mathrm{iSF}} \\
(\mathrm{ps})\end{array}$ & $\begin{array}{c}\tau_{\mathrm{rec}} \\
(\mathrm{ns})\end{array}$ \\
\hline $\mathbf{5}$ & 608 & 621 & 18 & --- & -- \\
\hline $\mathbf{1 b}$ & 608 & 625 & 3 & 13.8 & 79.6 \\
\hline $\mathbf{8}$ & 604 & 631 & 23 & --- & --- \\
\hline $\mathbf{2 b}$ & 605 & 625 & 2 & 135 & 100 \\
\hline
\end{tabular}

To determine if this was the operative fluorescence quenching process in these systems, transient absorption spectroscopy was utilized. Ultrafast transient absorption spectroscopy indicates that for both $\mathbf{1 b}$ and $\mathbf{2 b}$, an initial absorption feature reminiscent of the steady-state absorption spectrum appeared instantaneously upon excitation of the macrocycles by a pump laser light $(\lambda=560 \mathrm{~nm})$, which can be assigned to photoexcitation to the $S_{1}$ state (Figure $\mathbf{3 a}, \mathbf{b})$. The decay of this signal is concomitant with the rise of a new feature, assigned to the triplet state based on comparison to the spectral features of intersystem crossing observed in monomer 5 (Figure S25). The generation of this triplet excited state confirms the ability of both macrocycles to undergo intramolecular singlet fission. The time 
constant for singlet fission, $\tau_{\mathrm{iSF}}$, varies by over an order of magnitude between $\mathbf{1 b}$ (13.8 ps) and $\mathbf{2 b}$ (135.4 ps), implying that the larger degree of $\pi$-overlap between pentacene units in $\mathbf{1 b}$ enables faster, more efficient singlet fission (Figure 3b,d).

To determine whether this trend is mirrored in triplet lifetime $\left(\tau_{\text {rec }}\right)$, nanosecond transient absorption spectroscopy was utilized. Both macrocycles display biexponential decay, with the first component corresponding to the recombination of the correlated triplet pair $\left(\tau_{\text {rec }}\right)$, and the second to free triplet decay. Surprisingly, $\tau_{\text {rec }}$ does not change significantly between macrocycles within error (estimated to be $\mathbf{5 - 1 0 \% )}$ at $79.6 \mathrm{~ns}$ for $\mathbf{1 b}$ and $100 \mathrm{~ns}$ for $\mathbf{2 b}$. This runs counter to the commonly observed inverse relationship between $\tau_{\mathrm{iSF}}$ and $\tau_{\text {rec, }}$ where faster singlet fission is usually accompanied by shorter triplet lifetimes. This uncommon behavior is facilitated by the ability to access distinctly different through-space coupling motifs using these macrocyclic scaffolds, and suggests that they may be more broadly applicable for the independent modulation of these rates.

In conclusion, robust synthetic strategies for the synthesis of two unique pentacene-containing macrocycles on gram scale are demonstrated. These facile syntheses of a traditionally challenging class of compounds highlight the utility of zirconocene coupling in dynamic C-C bond forming routes to chromophore-containing macrocycles. Furthermore, the resulting macrocycles both undergo singlet fission in solution, displaying a desirable decoupling of SF rate and triplet lifetime. In the solid state, dimeric macrocycle $\mathbf{1 b}$ packs into columnar stacks that expand the macrocyclic cavity, introducing regular void space into the crystal lattice. This combination of unusual photophysical and supramolecular behavior provides strong preliminary evidence that shape-persistent macrocycles are desirable scaffolds for designing new classes of singlet fission materials. Current efforts involve co-crystallization of aromatic guests with dimeric macrocycle $\mathbf{1 b}$ to modulate its singlet fission dynamics in the solid state.

\section{ASSOCIATED CONTENT}

Supporting Information. The Supporting Information is available free of charge on the ACS Publications website at DOI: XXX

X-ray crystallographic data for S1 (CIF)

X-ray crystallographic data for 8 (CIF)

X-ray crystallographic data for $1 \mathrm{~b}(\mathrm{CIF})$

Experimental procedures and characterization data for all new compounds, including Schemes S1-S2, Figures S1-S30 and Tables S1-S3 (PDF)

\section{AUTHOR INFORMATION}

\section{Corresponding Author}

tdtilley@berkeley.edu

ORCID

Harrison M. Bergman: 0000-0001-6482-2837

Gavin R. Kiel: 0000-0001-6449-8547

Ryan J. Witzke: 0000-0003-1729-1636

Adam M. Schwartzberg: 0000-0001-6335-0719

Yi Liu: 0000-0002-3954-6102

T. Don Tilley: 0000-0002-6671-9099

\section{Funding Sources}

This work was funded by the National Science Foundation under Grant No. CHE-1708210.

\section{ACKNOWLEDGMENT}

We thank Dr Simon Teat (ALS, LBNL) for X-ray crystallography advice. Crystallographic analysis was performed at The Advanced Light Source, and transient absorption was performed at the Molecular Foundry, both of which are supported by the Director, Office of Science, Office of Basic Energy Sciences, of the U.S. Department of Energy under Contract No. DE-AC02-05CH11231. CIF files can also be obtained free of charge from the Cambridge Crystallographic Data Centre under reference numbers 2013263, 2013264, and 2013265.

\section{REFERENCES}

(1) Okamoto, T.; Bao, Z. Synthesis of Solution-Soluble Pentacene-Containing Conjugated Copolymers. J. Am. Chem. Soc. 2007, 129, 10308-10309.

(2) Park, S. K.; Jackson, T. N.; Anthony, J. E.; Mourey, D. A. High Mobility Solution Processed 6,13-Bis(TriisopropylSilylethynyl) Pentacene Organic Thin Film Transistors. Appl. Phys. Lett. 2007, 91, 063514.

(3) Smith, M. B.; Michl, J. Singlet Fission. Chem. Rev. 2010 , 110, 6891-6936.

(4) Smith, M. B.; Michl, J. Recent Advances in Singlet Fission. Annu. Rev. Phys. Chem. 2013, 64, 361-386.

(5) Rao, A.; Friend, R. H. Harnessing Singlet Exciton Fission to Break the Shockley-Queisser Limit. Nat. Rev. Mater. 2017, 2 , 17063.

(6) Walker, B. J.; Musser, A. J.; Beljonne, D.; Friend, R. H. Singlet Exciton Fission in Solution. Nat. Chem. 2013, 5, 10191024.

(7) Hetzer, C.; Guldi, D. M.; Tykwinski, R. R. Pentacene Dimers as a Critical Tool for the Investigation of Intramolecular Singlet Fission. Chem. - A Eur. J. 2018, 24, 8245-8257.

(8) Sanders, S. N.; Kumarasamy, E.; Pun, A. B.; Trinh, M. T.; Choi, B.; Xia, J.; Taffet, E. J.; Low, J. Z.; Miller, J. R.; Roy, X.; Zhu, X.-Y.; Steigerwald, M. L.; Sfeir, M. Y.; Campos, L. M. Quantitative Intramolecular Singlet Fission in Bipentacenes. J. 
Am. Chem. Soc. 2015, 137, 8965-8972.

(9) Zirzlmeier, J.; Lehnherr, D.; Coto, P. B.; Chernick, E. T.; Casillas, R; Basel, B. S.; Thoss, M.; Tykwinski, R. R.; Guldi, D. M. Singlet Fission in Pentacene Dimers. Proc. Natl. Acad. Sci. U. S. A. 2015, 112, 5325-5330.

(10) Sanders, S. N.; Kumarasamy, E.; Pun, A. B.; Steigerwald, M. L.; Sfeir, M. Y.; Campos, L. M. Intramolecular Singlet Fission in Oligoacene Heterodimers. Angew. Chemie. Int. Ed. 2016, 128, 3434-3438.

(11) Kumarasamy, E.; Sanders, S. N.; Tayebjee, M. J. Y.; Asadpoordarvish, A.; Hele, T. J. H.; Fuemmeler, E. G.; Pun, A. B.; Yablon, L. M.; Low, J. Z.; Paley, D. W.; Dean, J. C.; Choi, B.; Scholes, G. D.; Steigerwald, M. L.; Ananth, N.; Mccamey, D. R; Sfeir, M. Y.; Campos, L. M. Tuning Singlet Fission in $\Pi$-Bridge- $\pi$ Chromophores. J. Am. Chem. Soc. 2017, 139, 1248812494.

(12) Sanders, S. N.; Pun, A. B.; Parenti, K. R.; Kumarasamy, E.; Yablon, L. M.; Sfeir, M. Y.; Campos, L. M. Understanding the Bound Triplet-Pair State in Singlet Fission. Chem. 2019, 5, 1988-2005.

(13) Zirzlmeier, J.; Casillas, R.; Reddy, S. R.; Coto, P. B.; Lehnherr, D.; Chernick, E. T.; Papadopoulos, I.; Thoss, M.; Tykwinski, R. R; Guldi, D. M. Solution-Based Intramolecular Singlet Fission in Cross-Conjugated Pentacene Dimers. Nanoscale 2016, 8, 10113-10123.

(14) Basel, B. S.; Zirzlmeier, J.; Hetzer, C.; Reddy, S. R.; Phelan, B. T.; Krzyaniak, M. D.; Volland, M. K.; Coto, P. B.; Young, R. M.; Clark, T.; Thoss, M.; Tykwinski, R. R; Wasielewski, M. R.; Guldi, D. M. Evidence for Charge-Transfer Mediation in the Primary Events of Singlet Fission in a Weakly Coupled Pentacene Dimer. Chem 2018, 4, 1092-1111.

(15) Papadopoulos, I.; Zirzlmeier, J.; Hetzer, C.; Bae, Y. J.; Krzyaniak, M. D.; Wasielewski, M. R.; Clark, T.; Tykwinski, R. R.; Guldi, D. M. Varying the Interpentacene Electronic Coupling to Tune Singlet Fission. J. Am. Chem. Soc. 2019, 141, 61916203.

(16) Hetzer, C.; Basel, B. S.; Kopp, S. M.; Hampel, F.; White, F. J.; Clark, T.; Guldi, D. M.; Tykwinski, R. R. Chromophore Multiplication To Enable Exciton Delocalization and Triplet Diffusion Following Singlet Fission in Tetrameric Pentacene. Angew. Chemie Int. Ed. 2019, 58, 15263-15267.

(17) Casillas, R.; Adam, M.; Coto, P. B.; Waterloo, A. R.; Zirzlmeier, J.; Reddy, S. R.; Hampel, F.; McDonald, R; Tykwinski, R. R.; Thoss, M.; Guldi, D. M. Intermolecular Singlet Fission in Unsymmetrical Derivatives of Pentacene in Solution. Adv. Energy Mater. 2019, 9, 1802221.

(18) Basel, B. S.; Young, R. M.; Krzyaniak, M. D.; Papadopoulos, I.; Hetzer, C.; Gao, Y.; La Porte, N. T.; Phelan, B. T.; Clark, T.; Tykwinski, R. R.; Wasielewski, M. R.; Guldi, D. M. Influence of the Heavy-Atom Effect on Singlet Fission: A Study of Platinum-Bridged Pentacene Dimers. Chem. Sci. 2019, 10, 11130-11140.

(19) Chen, M.; Bae, Y. J.; Mauck, C. M.; Mandal, A.; Young, R. M.; Wasielewski, M. R. Singlet Fission in Covalent Terrylenediimide Dimers: Probing the Nature of the Multiexciton State Using Femtosecond Mid-Infrared Spectroscopy. J. Am. Chem. Soc. 2018, 140, 9184-9192.
(20) Mandal, A.; Chen, M.; Foszcz, E. D.; Schultz, J. D.; Kearns, N. M.; Young, R. M.; Zanni, M. T.; Wasielewski, M. R. Two-Dimensional Electronic Spectroscopy Reveals Excitation Energy-Dependent State Mixing during Singlet Fission in a Terrylenediimide Dimer. J. Am. Chem. Soc. 2018, 140, 1790717914.

(21) Margulies, E. A.; Miller, C. E.; Wu, Y.; Ma, L.; Schatz, G. C.; Young, R. M.; Wasielewski, M. R. Enabling Singlet Fission by Controlling Intramolecular Charge Transfer in $\pi$-Stacked Covalent Terrylenediimide Dimers. Nat. Chem. 2016, 8, 11201125 .

(22) Papadopoulos, I.; Gao, Y.; Hetzer, C.; Tykwinski, R. R.; Guldi, D. M. Singlet Fission in Enantiomerically Pure Pentacene Dimers. ChemPhotoChem. 2020, 4, 1-8.

(23) Pun, A. B.; Asadpoordarvish, A.; Kumarasamy, E.; Tayebjee, M. J. Y.; Niesner, D.; McCamey, D. R.; Sanders, S. N.; Campos, L. M.; Sfeir, M. Y. Ultra-Fast Intramolecular Singlet Fission to Persistent Multiexcitons by Molecular Design. Nat. Chem. 2019, 11, 821-828.

(24) Pensack, R. D.; Tilley, A. J.; Grieco, C.; Purdum, G. E.; Ostroumov, E. E.; Granger, D. B.; Oblinsky, D. G.; Dean, J. C.; Doucette, G. S.; Asbury, J. B.; Loo, Y.-L.; Seferos, D. S.; Anthony, J. E.; Scholes, G. D. Striking the Right Balance of Intermolecular Coupling for High-Efficiency Singlet Fission. Chem. Sci. 2018, 9, 6240-6259.

(25) Yost, S. R.; Lee, J.; Wilson, M. W. B.; Wu, T.; McMahon, D. P.; Parkhurst, R. R; Thompson, N. J.; Congreve, D. N.; Rao, A.; Johnson, K.; Sfeir, M. Y.; Bawendi, M. G.; Swager, T. M.; Friend, R. H.; Baldo, M. A.; Van Voorhis, T. A Transferable Model for Singlet-Fission Kinetics. Nat. Chem. 2014, 6, 492 497.

(26) Masoomi-Godarzi, S.; Liu, M.; Tachibana, Y.; Mitchell, V. D.; Goerigk, L.; Ghiggino, K. P.; Smith, T. A.; Jones, D. J. Liquid Crystallinity as a Self-Assembly Motif for HighEfficiency, Solution-Processed, Solid-State Singlet Fission Materials. Adv. Energy Mater. 2019, 9, 1901069.

(27) O’Neill, M.; Kelly, S. M. Liquid Crystals for Charge Transport, Luminescence, and Photonics. Adv. Mater. 2003, 15 , 1135-1146.

(28) Lu, H.; Chen, X.; Anthony, J. E.; Johnson, J. C.; Beard, M. C. Sensitizing Singlet Fission with Perovskite Nanocrystals. J. Am. Chem. Soc. 2019, 141, 4919-4927.

(29) Saegusa, T.; Sakai, H.; Nagashima, H.; Kobori, Y.; Tkachenko, N. V.; Hasobe, T. Controlled Orientations of Neighboring Tetracene Units by Mixed Self-Assembled Monolayers on Gold Nanoclusters for High-Yield and LongLived Triplet Excited States through Singlet Fission. J. Am. Chem. Soc. 2019, 141, 14720-14727.

(30) Ball, M.; Zhong, Y.; Fowler, B.; Zhang, B.; Li, P.; Etkin, G.; Paley, D. W.; Decatur, J.; Dalsania, A. K.; Li, H.; Xiao, S.; Ng, F.; Steigerwald, M. L.; Nuckolls, C. Macrocyclization in the Design of Organic N-Type Electronic Materials. J. Am. Chem. Soc. 2016, 138, 12861-12867.

(31) Ball, M. L.; Zhang, B.; Xu, Q.; Paley, D. W.; Ritter, V. C.; $\mathrm{Ng}$, F.; Steigerwald, M. L.; Nuckolls, C. Influence of Molecular Conformation on Electron Transport in Giant, Conjugated Macrocycles. J. Am. Chem. Soc. 2018, 140, 10135-10139.

(32) Gong, X.; Young, R. M.; Hartlieb, K. J.; Miller, C.; Wu, Y.; 
Xiao, H.; Li, P.; Hafezi, N.; Zhou, J.; Ma, L.; Cheng, T.; Goddard, W. A.; Farha, O. K.; Hupp, J. T.; Wasielewski, M. R; Stoddart, J. F. Intramolecular Energy and Electron Transfer within a Diazaperopyrenium-Based Cyclophane. J. Am. Chem. Soc. 2017, 139, 4107-4116.

(33) Kim, D. J.; Hermann, K. R.; Prokofjevs, A.; Otley, M. T.; Pezzato, C.; Owczarek, M.; Stoddart, J. F. Redox-Active Macrocycles for Organic Rechargeable Batteries. J. Am. Chem. Soc. 2017, 139, 6635-6643.

(34) Beldjoudi, Y.; Narayanan, A.; Roy, I.; Pearson, T. J.; Cetin, M. M.; Nguyen, M. T.; Krzyaniak, M. D.; Alsubaie, F. M.; Wasielewski, M. R.; Stupp, S. I.; Stoddart, J. F. Supramolecular Tessellations by a Rigid Naphthalene Diimide Triangle. J. Am. Chem. Soc. 2019, 141, 17783-17795.

(35) Mohan Nalluri, S. K.; Zhou, J.; Cheng, T.; Liu, Z.; Nguyen, M. T.; Chen, T.; Patel, H. A.; Krzyaniak, M. D.; Goddard, W. A.; Wasielewski, M. R.; Stoddart, J. F. Discrete Dimers of Redox-Active and Fluorescent Perylene DiimideBased Rigid Isosceles Triangles in the Solid State. J. Am. Chem. Soc. 2019, 141, 1290-1303.

(36) Ball, M.; Zhang, B.; Zhong, Y.; Fowler, B.; Xiao, S.; Ng, F.; Steigerwald, M.; Nuckolls, C. Conjugated Macrocycles in Organic Electronics. Acc. Chem. Res. 2019, 52, 1068-1078.

(37) Bula, R.; Fingerle, M.; Ruff, A.; Speiser, B.; MaichleMössmer, C.; Bettinger, H. F. Anti -[2.2](1,4)Pentacenophane: A Covalently Coupled Pentacene Dimer. Angew. Chemie Int. Ed. 2013, 52, 11647-11650.

(38) Kuroda, K.; Yazaki, K.; Tanaka, Y.; Akita, M.; Sakai, H.; Hasobe, T.; Tkachenko, N. V.; Yoshizawa, M. A PentaceneBased Nanotube Displaying Enriched Electrochemical and Photochemical Activities. Angew. Chemie Int. Ed. 2019, 58, 1115-1119.

(39) Gessner, V. H.; Tannaci, J. F.; Miller, A. D.; Tilley, T. D. Assembly of Macrocycles by Zirconocene-Mediated, Reversible
Carbon-Carbon Bond Formation. Acc. Chem. Res. 2011, 44, 435-446.

(40) Ge, P.-H.; Fu, W.; Herrmann, W. A.; Herdtweck, E.; Campana, C.; Adams, R. D.; Bunz, U. H. F. Structural Characterization of a Cyclohexameric metaPhenyleneethynylene Made by Alkyne Metathesis with In Situ Catalysts. Angew. Chemie. 2000, 39, 3607-3610.

(41) Miljaniae, O. Š.; Peter, K.; Vollhardt, C.; Whitener, G. D. An Alkyne Metathesis-Based Route to OrthoDehydrobenzannulenes. Synlett 2003, 1, 29-34.

(42) Zhang, W.; Moore, J. S. Arylene Ethynylene Macrocycles Prepared by Precipitation-Driven Alkyne Metathesis. J. Am. Chem. Soc. 2004, 126, 12796.

(43) Jin, Y.; Zhang, A.; Huang, Y.; Zhang, W. Shape-Persistent Arylenevinylene Macrocycles (AVMs) Prepared via Acyclic Diene Metathesis Macrocyclization (ADMAC). Chem. Commun. 2010, 46, 8258-8260.

(44) Gessner, V. H.; Tilley, T. D. Diphenylanthracene Macrocylces from Reductive Zirconocene Coupling: On the Edge of Steric Overload. Org. Lett. 2011 , 13, 1154-1157.

(45) Rosenthal, U.; Ohff, A.; Baumann, W.; Tillack, A.; Gurls, H.; Burlakov, V. V.; Shur, V. B. Struktur, Eigenschaften Und NMR-Spektroskopische Charakterisierung von $\mathrm{Cp}_{2} \mathrm{Zr}$ (Pyridin) $\left(\mathrm{Me}_{3} \mathrm{SiC} \equiv \mathrm{CSiMe}_{3}\right)$.Zeitschrift fur Anorg. und Allg. Chemie. 1995, 621, 77-83.

(46) Nitschke, J. R.; Zurcher, S.; Tilley, T. D. New Zirconocene-Coupling Route to Large, Functionalized Macrocycles. J. Am. Chem. Soc. 2000, 122, 10345-10352.

(47) Montgomery, H. C.; Schön, J. H.; Kloc, C.; Batlogg, B. Functionalized Pentacene: Improved Electronic Properties from Control of Solid-State Order. J. Am. Chem. Soc 2001, 123, 9482 9483. 


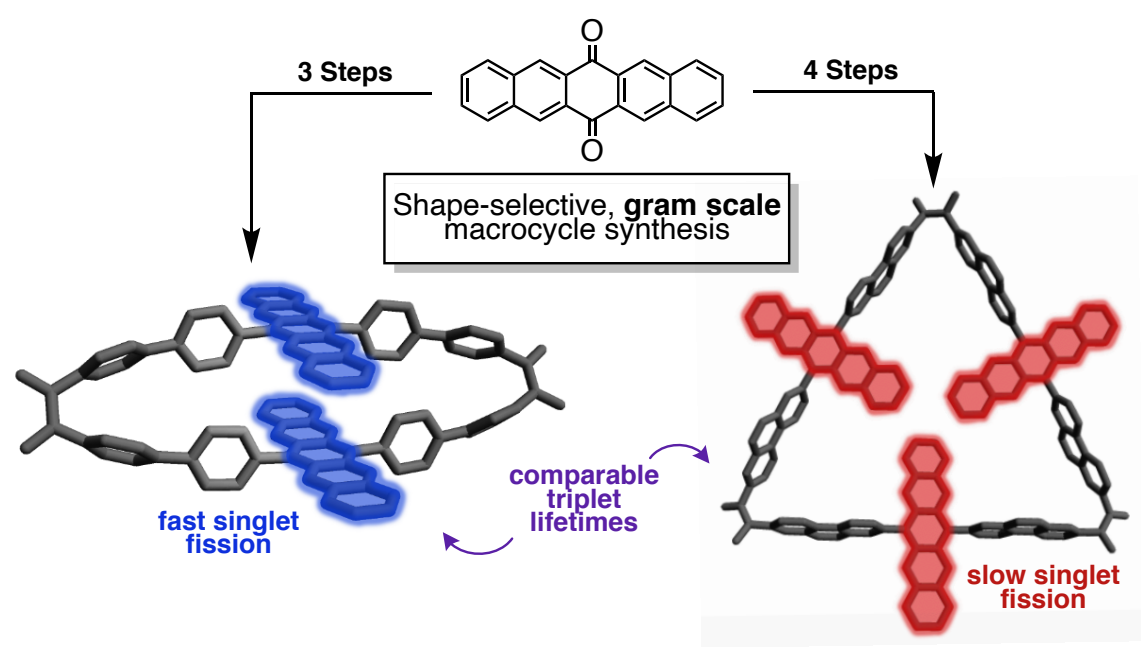

